\title{
The 7-valent pneumococcal conjugate vaccine elicits cross-functional opsonophagocytic killing responses to Streptococcus pneumoniae serotype 6D in children
}

Hyunju Lee ${ }^{1,2}$, Jung Hwa Cha', Moon H Nahm³ ${ }^{3}$, Robert L Burton ${ }^{3}$ and Kyung-Hyo Kim,2*

\begin{abstract}
Background: We investigated the immune response to serogroup 6 with the opsonophagocytic killing assay (OPKA) in children aged 12-23 months of age after immunization with the 7-valent pneumococcal conjugate vaccine (PCV7) containing serotype 6B.

Methods: Blood samples were obtained from 59 children who had blood sampling for medical examination. Immunization status against PCV7 was confirmed by immunization records and samples were categorized according to immunization status into a booster, primary, or control group. The OPKA was performed for serotypes 6A, 6B, 6C, and 6D.

Results: Subjects with no previous PCV7 immunization history showed opsonic activity for serogroup 6 in 5-30\% (according to serotype). In subjects vaccinated with a 3-dose primary series, 81\% showed opsonic activity for serotypes 6B and 6D, and 29\% showed opsonic activity for serotypes 6A and 6C. Among subjects vaccinated with a booster dose, all subjects had opsonic activity against serotype 6B. Subjects in the booster group with opsonic activity against serotypes $6 \mathrm{~A}, 6 \mathrm{C}$, and $6 \mathrm{D}$ were $100 \%, 78 \%$, and $89 \%$, respectively.

Conclusions: In subjects aged 12-23 months, an immune response is elicited after a primary series of immunizations with PCV7 for serotypes $6 \mathrm{~B}$ and $6 \mathrm{D}$, and a booster dose enhances a cross reactive immune response against serotypes $6 \mathrm{~A}, 6 \mathrm{C}$ and $6 \mathrm{D}$.
\end{abstract}

Keywords: Streptococcus pneumoniae, Heptavalent pneumococcal conjugate vaccine, Pneumococcal polysaccharide type 6

\section{Background}

Streptococcus pneumoniae is a major cause of meningitis, pneumonia, and bacteremia in children and adults throughout the world. It is also a major cause of acute otitis media and sinusitis. With the introduction of the 7-valent pneumococcal conjugate vaccine (PCV7, Prevenar, Pfizer Inc, Philadelphia, PA), a substantial decrease in invasive pneumococcal disease (IPD) due to serotypes included in the vaccine was seen among children and adults [1] since

\footnotetext{
* Correspondence: kaykim@ewha.ac.kr

'Department of Pediatrics, Ewha Womans University School of Medicine, Seoul, Korea

${ }^{2}$ Center for Vaccine Evaluation and Study, Ewha Medical Research Institute, Ewha Womans University, Seoul, Korea

Full list of author information is available at the end of the article
}

protection against S. pneumoniae is a serotype-specific immune response [2].

Up to several years ago, only serotypes $6 \mathrm{~A}$ and $6 \mathrm{~B}$ were grouped into serogroup 6 due to similarity in both chemical structure and serological properties of their capsular polysaccharides. Following the recent discovery of two new members, serogroup 6 now consists of 4 serotypes: 6A, 6B, 6C, and 6D [3,4]. Although S. pneumoniae serotype 6C is quite prevalent throughout the world, serotype $6 \mathrm{D}$ isolates are relatively rare in many countries [5-14]. For instance, the US CDC identified only two 6D isolates in its extensive multi-year surveys [15]. This rare finding of 6D in the US may be due to adequate cross-protection against $6 \mathrm{D}$ by 
PCV7, which contains serotype 6B PS that is similar to $6 \mathrm{D}$ PS in structure.

In contrast to the US, several epidemiologic studies in Korea reported high prevalence of serotype 6D, which accounts for up to $5-10 \%$ of serogroup 6 isolates $[16,17]$. They are found both among adults and children. This unusually high prevalence of $6 \mathrm{D}$ is unexpected since PCV7 is also widely used in Korea. Therefore it is possible that PCV7 does not elicit cross-protective antibodies against serotype 6D among Korean children. To investigate this possibility, we examined the immune response to serotypes $6 \mathrm{~A}, 6 \mathrm{C}$, and $6 \mathrm{D}$, as well as $6 \mathrm{~B}$, with the opsonophagocytic killing assay (OPKA) in children aged 12-23 months of age after vaccination with PCV7.

\section{Methods}

\section{Subjects}

Subjects included in this study were children aged 12-23 months who had blood sampling for medical examination. Blood samples were obtained after informed consent. Immunization status against PCV7 was confirmed by immunization records and samples were categorized according to immunization status. Subjects with 3 primary doses and 1 booster dose of PCV7 were assigned to the booster group, subjects with 3 primary doses before 12 months of age were assigned to the primary group, and subjects with no vaccination history of PCV7 were assigned to the control group. Samples from 45 subjects who visited Kangnam CHA Medical Center from September to December 2006 have been described previously [18]. Serum samples from 14 subjects were additionally collected at Ewha Womans University Mokdong Hospital from November 2007 to February 2008. Children with underlying immunodeficiency disorders or a history of blood transfusion, immunoglobulin, or systemic steroid medication were excluded from the study. The study protocol was approved by the Institutional Review Board at Kangnam CHA Medical Center and Ewha Womans University Mokdong Hospital and was conducted in accordance with the Declaration of Helsinki and Good Clinical Practice guidelines. Informed written consent was obtained from all parents or legal guardians following a detailed explanation of the study.

\section{Opsonophagocytic killing assay}

The opsonic indices (OI) of the samples were determined using the OPKA as previously described [19-23]. Target strains TREP6A, SPEC6B, SPEC6C, and SPEC6D (expressing capsule types $6 \mathrm{~A}, 6 \mathrm{~B}, 6 \mathrm{C}$, and $6 \mathrm{D}$, respectively) were derived from wild-type strains EF6796, BG25-9, BGO-2197, and MNZ920, respectively, and have been described previously $[19,24]$. Opsonic indices were defined as the serum dilution that kills $50 \%$ of bacteria and were determined by linear interpolation. In this study, all sera were diluted 5- fold before the assay due to limited amounts of sera. Because all sera were diluted 5-fold during the assay, the limit of detection was 20. A detailed protocol is posted on a website (www.vaccine.uab.edu).

\section{Statistical analysis}

The analyses of serum antibody OI were based on logarithms of the OIs of all subjects. Geometric mean opsonic indices (GMI) were evaluated and two-sided 95\% confidence intervals were determined for each pneumococcal serotype. Serum samples with OIs $<20$ were assigned a value of 10 for analysis purposes. The proportions of subjects achieving anti-pneumococcal OI $\geq 20$ were determined. Reverse cumulative distribution curves were used to display percentages of children that achieved different $\mathrm{OI}$ to each of the 4 pneumococcal serotypes, $6 \mathrm{~A}, 6 \mathrm{~B}, 6 \mathrm{C}$, and $6 \mathrm{D}$.

\section{Results}

\section{Characteristics of the subjects}

A total of 59 subjects were included in the study. There were 18 subjects, 21 subjects, and 20 subjects in the booster, primary, and control group, respectively. The mean age of subjects in the booster group was 18.8 months (range 16-23 months) with a mean interval between last vaccination and sampling of 3.3 months (range 1-7 months). The primary group consisted of subjects with a mean age of 13.9 months (range 12-16 months) and the mean interval between the last vaccination and sampling was 7.2 months (range 4-9 months). The subjects in the control group were of a mean age of 14.5 months (range 12-16 months).

\section{Immune response to serogroup 6}

The GMI for each serotype in serogroup 6 for each group of subjects is shown in Table 1. GMI of the booster group was higher compared with the primary and control groups for all 4 serotypes tested (Figure 1). When comparing the

Table 1 Geometric mean indices of serogroup 6 in response to $\mathrm{PCV} 7$ vaccine according to vaccination status

\begin{tabular}{lllll}
\hline & & Booster (N=18) & Primary (N=21) & Control $(\mathbf{N}=\mathbf{2 0})$ \\
\hline $6 \mathrm{~B}$ & $\mathrm{GMl}$ & 5,948 & $123^{*},+$ & $19^{*}$ \\
& $95 \% \mathrm{Cl}$ & $(2,969-11,917)$ & $(55-263)$ & $(9-47)$ \\
$6 \mathrm{~A}$ & $\mathrm{GMl}$ & 2,065 & $29^{*}$ & $23^{*}$ \\
& $95 \% \mathrm{Cl}$ & $(971-4,395)$ & $(12-67)$ & $(9-68)$ \\
$6 \mathrm{C}$ & $\mathrm{GMl}$ & 808 & $30^{*}$ & 13 \\
& $95 \% \mathrm{Cl}$ & $(224-2,913)$ & $(12-63)$ & $(8-24)$ \\
$6 \mathrm{C}$ & $\mathrm{GMl}$ & 2,323 & $207^{*}$ & $79^{*}$ \\
& $95 \% \mathrm{Cl}$ & $(683-7,988)$ & $(57-357)$ & $(21-476)$
\end{tabular}

${ }^{*} P<0.05$ compared with booster, ${ }^{\dagger} P<0.05$ compared with control. PCV7; 7-valent pneumococcal conjugate vaccine, $\mathrm{N}$; number, GMl; geometric mean index, $\mathrm{Cl}$; confidence interval. 


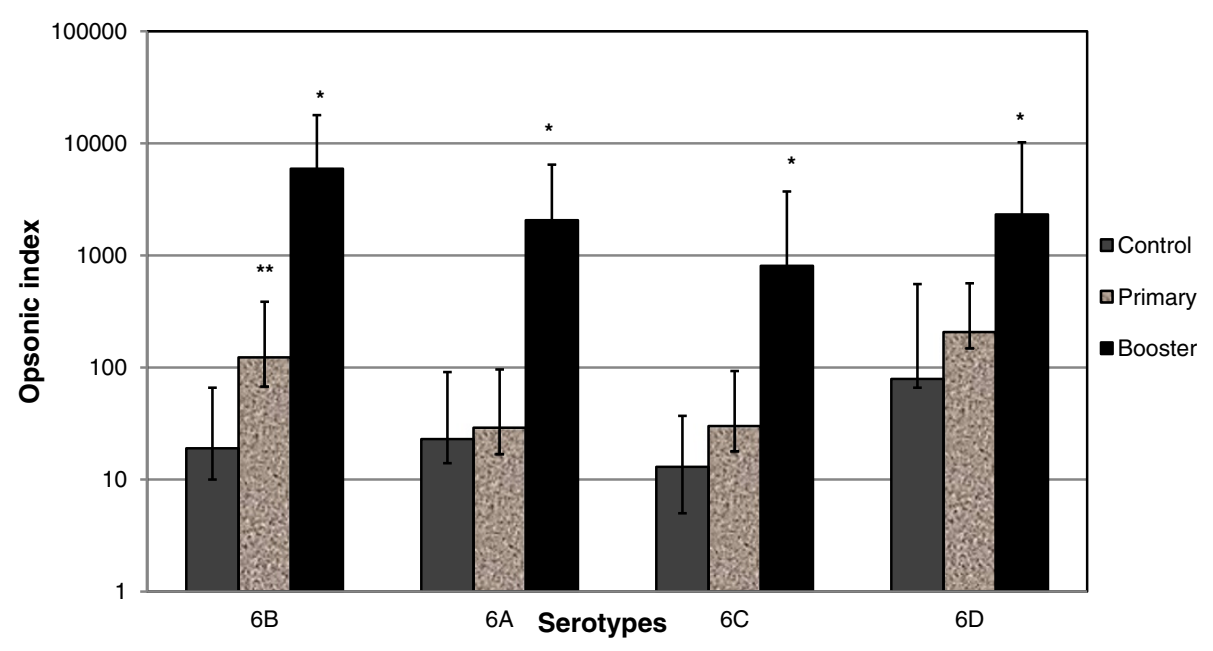

Figure 1 Geometric mean opsonic indices of serogroup 6 in response to PCV7 according to vaccination status. ${ }^{*} P<0.05$, Booster compared with primary and control groups; ${ }^{*} P<0.05$, Primary compared with control group PCV7; 7-valent pneumococcal conjugate vaccine.

primary and control groups, GMI was higher for serotype $6 \mathrm{~B}(p<0.05)$ in the primary group, however there was no difference between these groups for serotype $6 \mathrm{~A}, 6 \mathrm{C}$, and $6 \mathrm{D}$.

In the control group, $5-30 \%$ of the subjects had an OI $>=20$, depending on serotype (Figure 2 and data not shown). In subjects who had previously been vaccinated with PCV7 as a primary series, and have not yet received the booster dose, $81 \%$ of the subjects had an OI $\geq 20$ for serotypes $6 \mathrm{~B}$ and $6 \mathrm{D}$. However in the same subjects, only $29 \%$ had an OI $\geq 20$ for serotypes $6 \mathrm{~A}$ and $6 \mathrm{C}$. After a booster dose, all subjects had OIs $\geq 20$ for serotype $6 \mathrm{~B}$ and $6 \mathrm{~A}$, while $89 \%$ and $78 \%$ had OIs $\geq 20$ for serotypes $6 \mathrm{D} 6 \mathrm{C}$, respectively.

\section{Discussion}

Our study shows that PCV7 can elicit high levels of antibodies cross-reacting with serotypes $6 \mathrm{~A}, 6 \mathrm{C}$, and $6 \mathrm{D}$ in children 12-23 months of age. For serotype 6D, opsonic activity was demonstrable in $81 \%$ of the primary group and $89 \%$ of the booster group in these children. These high antibody responses are comparable with that of serotype $6 \mathrm{~B}$ and actually higher than those for serotypes $6 \mathrm{~A}$ and $6 \mathrm{C}$. This finding is consistent with the paucity of 6D isolates found in the US, where PCV7 is used extensively. In contrast, 6D isolates are relatively more prevalent in Korea. Although there are no nationwide studies in Korea, according to a study of isolates collected from $1991-2008,10.4 \%$ of serogroup 6 isolates were found to

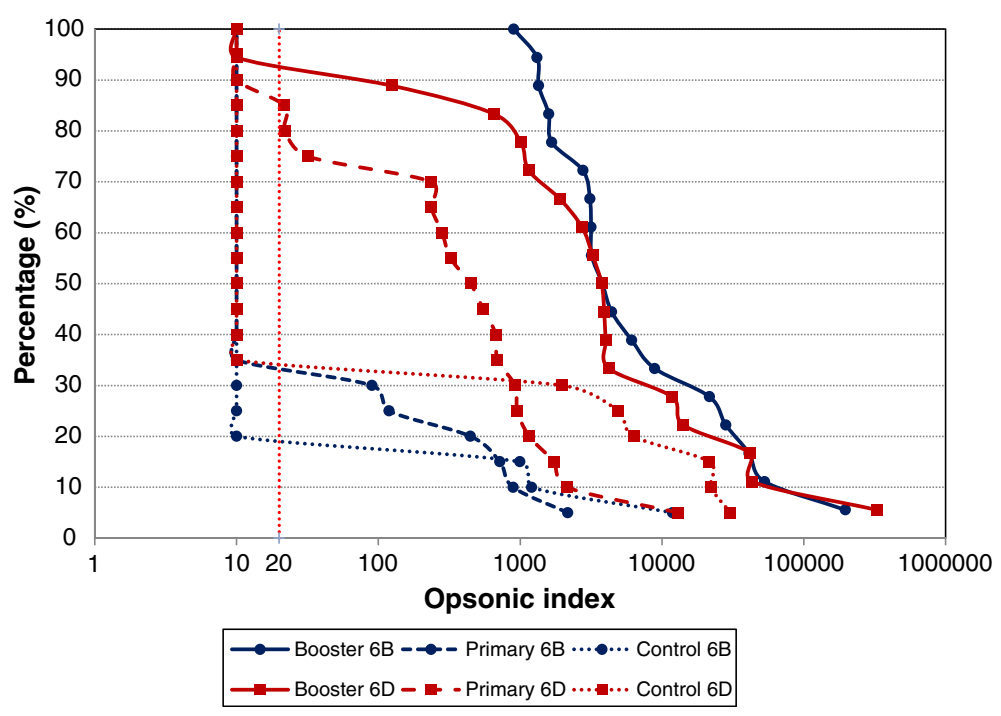

Figure 2 Reverse cumulative distribution curves of opsonic indices for serotypes 6B and 6D in booster, primary and control groups. 
be serotype 6D [17]. A second study of isolates collected from 1997-2009 seemed to show an increase in 6D isolates in recent years [25]. However these studies were done on various populations of different ages. Therefore, we cannot directly determine the effect of PCV7 on serotype $6 \mathrm{D}$. Further studies are needed to determine the effect of PCV7 on the epidemiology of 6D. If an increase in serotype 6D prevalence is real, it would be interesting to assess the OPA activity against 6D using isolates from Korea as target strains, to verify the high cross-protection immune response that was detected in this study.

In this study, $29 \%$ of the subjects in the primary group had a detectable OI for serotype 6C. This is consistent with the results of a previous study which found a seropositivity rate of $39 \%$ in infants following a 3-dose primary series (unpublished data). However, in the current study, $78 \%$ of subjects had a detectable OI for $6 \mathrm{C}$ in the booster group. Thus, the booster dose seems to significantly enhance cross-protection against $6 \mathrm{C}$.

The seropositivity rate for serotype $6 \mathrm{C}$ in the primary group was relatively low compared with serotype 6D ( $29 \%$ vs $81 \%$ ). This finding supports the epidemiologic phenomenon seen in 2004-2008 where an increase in invasive pneumococcal diseases due to serotype $6 \mathrm{C}$ was seen during a vaccine shortage [26]. Due to this vaccine shortage, many children were not able to receive a booster vaccination in 2004. With the absence of the booster dose, cross-protection was not sufficient for vaccine related serotypes $[27,28]$.

The results of this study emphasize the importance of a booster dose for better protection of disease caused by both vaccine serotypes and vaccine-related serotypes. Compared to the control group which had no history of PCV7 vaccination, the immune responses to all serogroup 6 members were higher in primary group and highest in booster group. Infants are restricted in their ability to make diverse antibody variable regions and may produce antibodies with low avidity to crossreactive serotypes [29]. However, it is known that multiple immunizations improve antibody maturation through somatic mutation resulting in high affinity antibody [30,31]. Our results of the cross-protective immune response according to vaccination dosages support this finding.

There are limitations in this study. OPKA threshold for protection is identified as $\mathrm{OI} \geq 8$. Although there is controversy as to whether it is appropriate to apply a consistent value for all serotypes, Henckaerts et al. reported in a validation study of the OPKA, that OPKA sero-positivity $(\mathrm{OI} \geq 8)$ correlated well with IPD effectiveness [32]. Since the threshold for OPKA was an OI $\geq 20$ in this study, the results may be rather underestimated. Also, there were differences in age groups, where the mean age of children in the booster group was higher than the primary or control groups. Although none of the infants in the study had documented history of pneumococcal disease, this could have an effect on the antibody titers between groups, related to increase in natural exposure to pneumococcus with increase in age.

Compared to published data of the US, European, or Taiwanese infants, Korean infants had higher postvaccination antibody titer to all serotypes in PCV7 [33]. Therefore further studies are needed in different population or ethnic groups. Moreover, this study is based on the immune response of PCV7. Recently conjugate vaccines containing more serotypes and different conjugate proteins have been introduced into the market $[34,35]$. The results of this study cannot be directly applied to these new vaccines, and further study is needed for different vaccine formulations. However PCV7 is still used in many countries. In this regard, the immune responses of PCV7 are of importance.

\section{Conclusion}

We found that in subjects aged 12-23 months, an immune response is elicited after a primary series of immunizations with PCV7 for serotypes $6 \mathrm{~B}$ and $6 \mathrm{D}$ and a booster dose enhances cross reactive antibody levels against serotypes $6 \mathrm{~A}, 6 \mathrm{C}$, and $6 \mathrm{D}$.

\section{Abbreviations \\ OPKA: Opsonophagocytic killing assay; PCV7: 7-valent pneumococcal conjugate vaccine; IPD: Invasive pneumococcal disease; Ol: Opsonic index GMI: Geometric mean opsonic index; PS: Polysaccharide.}

\section{Competing interests}

This study was funded by RP-Grant 2011 of Ewha Womans University to HL and KHK and by the Korea Food and Drug Administration (11172KFDA360) to KHK. University of Alabama at Birmingham (UAB) owns intellectual property rights on the various reagents used for pneumococcal vaccine studies, and $\mathrm{MHN}$ and RLB are UAB employees.

\section{Authors' contributions}

Lee $\mathrm{H}$ managed the data and wrote the manuscript, Cha JW helped with statistical analysis, Burton RL carried out the immunoassays, Nahm $\mathrm{MH}$ participated in the preparation of the manuscript, and Kim $\mathrm{KH}$ participated in the design of the study and conceived of the study. All authors read and approved the final manuscript.

\section{Author details}

${ }^{1}$ Department of Pediatrics, Ewha Womans University School of Medicine, Seoul, Korea. ${ }^{2}$ Center for Vaccine Evaluation and Study, Ewha Medical Research Institute, Ewha Womans University, Seoul, Korea. ${ }^{3}$ Departments of Pathology and Microbiology, University of Alabama at Birmingham, Birmingham, Alabama 35294, USA.

Received: 27 March 2013 Accepted: 3 October 2013

Published: 10 October 2013

\section{References}

1. Center for Disease Control and Prevention: Direct and indirect effects of routine vaccination of children with 7-valent pneumococcal conjugate vaccine on incidence of invasive pneumococcal disease--United States, 1998-2003. MMWR Morb Mortal Wkly Rep 2005, 54:893-897. 
2. Hsu KK, Shea KM, Stevenson AE, Pelton SI: Changing serotypes causing childhood invasive pneumococcal disease: Massachusetts, 2001-2007. Pediatr Infect Dis J 2010, 29:289-293.

3. Bratcher PE, Park IH, Oliver MB, Hortal M, Camilli R, Hollingshead SK, Camou T, Nahm MH: Evolution of the capsular gene locus of Streptococcus pneumoniae serogroup 6. Microbiology 2011, 157:189-198.

4. Park IH, Moore MR, Treanor JJ, Pelton SI, Pilishvili T, Beall B, Shelly MA, Mahon BE, Nahm MH, Active Bacterial Core Surveillance Team: Differential effects of pneumococcal vaccines against serotypes $6 \mathrm{~A}$ and $6 \mathrm{C}$. J Infect Dis 2008, 198:1818-1822

5. Bratcher PE, Park IH, Hollingshead SK, Nahm MH: Production of a unique pneumococcal capsule serotype belonging to serogroup 6 .

Microbiology 2009, 155:576-583.

6. Bratcher $\mathrm{PE}$, Kim KH, King JH, Hong JY, Moon MH: Identification of natural pneumococcal isolates expressing serotype 6D by genetic, biochemical and serological characterization. Microbiology 2010, 156:555-560.

7. Chang B, Otsuka T, Iwaya A, Okazaki M, Matsunaga S, Wada A: Isolation of Streptococcus pneumoniae serotypes $6 C$ and $6 D$ from the nasopharyngeal mucosa of healthy Japanese children. Jpn J Infect Dis 2010, 63:381-383.

8. Ho P-L, Ang I, Chow K-H, Lai EL, Chiu SS: The prevalence and characteristics of Streptococcus pneumoniae isolates expressing serotypes $6 \mathrm{C}$ and $6 \mathrm{D}$ in Hong Kong prior to the introduction of the 7-valent pneumococcal conjugate vaccine. Diagn Microbiol Infect Dis 2010, 68:439-444

9. Kuch A, Sadowy E, Skoczyńska A, Hryniewicz W: First report of Streptococcus pneumoniae serotype 6D isolates from invasive infections. Vaccine 2010, 28:6406-6407.

10. Mercado E, Srinivansan V, Hawkins P, Chochua S, Ochoa T, Beall B, McGee L: First report of Streptococcus pneumoniae serotype 6D in South America. J Clin Microbiol 2011, 49:2080-2081

11. Nahm MH, Oliver MB, Siira L, Kaijalainen $T$, Lambertsen $L M$, Virolainen $A$ : A report of Streptococcus pneumoniae serotype 6D in Europe. J Med Microbiol 2011, 60:46-48.

12. Oftadeh S, Satzke C, Gilbert GL: Identification of newly described Streptococcus pneumoniae serotype 6D by use of the Quellung reaction and PCR. J Clin Microbiol 2010, 48:3378-3379.

13. Yao KH, Liu ZJ, Yu JG, Yu SJ, Yuan L, Nahm MH, Yang YH: Type distribution of serogroup 6 Streptococcus pneumoniae and molecular epidemiology of newly identified serotypes 6C and 6D in China. Diagn Microbiol Infect Dis 2011, 70:291-298.

14. Adam HJ, Karlowsky JA, Nichol KA, Gilmour MW, Hoban DH, Embree J, Zhanel GG: Baseline epidemiology of Streptococcus pneumoniae serotypes in Canada prior to the introduction of the 13-valent pneumococcal vaccine. Microb Drug Resist 2012, 18:176-182.

15. Massire C, Gertz RE Jr, Svoboda P, Levert K, Reed MS, Pohl J, Kreft R, Li F, White N, Ranken R, Blyn LB, Ecker DJ, Sampath R, Beall B: Concurrent serotyping and genotyping of pneumococci by use of PCR and electrospray ionization mass spectrometry. Clin Microbiol 2012, 50:2018-2025.

16. Baek JY, Ko KS, Song JH: Streptococcus pneumoniae serotype 6D crossreacting with serotype $6 \mathrm{~A}, 6 \mathrm{~B}$, and $6 \mathrm{C}$ factor sera. J Clin Microbiol 2011, 49:765-766

17. Choi EH, Lee HJ, Cho EY, Oh CE, Eun BW, Lee J, Kim MJ: Prevalence and genetic structures of Streptococcus pneumoniae serotype 6D, South Korea. Emerg Infect Dis 2010, 16:1751-1753.

18. Park SE, Lee H, Lim SY, Kim KH: Immunogenicity of 7-valent pneumococcal conjugate vaccine related to booster immunization in Korean children. Korean J Pediatr 2008, 51:622-628.

19. Burton RL, Nahm MH: Development and validation of a fourfold multiplexed opsonization assay (MOPA4) for pneumococcal antibodies. Clin Vaccine Immunol 2006, 13:1004-1009.

20. Wang D, Burton RL, Nahm MH, Soong SJ: A four-parameter logistic model for estimating titers of functional multiplexed pneumococcal opsonophagocytic killing assay. J Biopharm Stat 2008, 18:307-325.

21. Romero-Steiner $\mathrm{S}$, Frasch CE, Carlone G, Fleck RA, Goldblatt D, Nahm MH: Use of opsonophagocytosis for serological evaluation of pneumococcal vaccines. Clin Vaccine Immunol 2006, 13:165-169.

22. Romero-Steiner S, Libutti D, Pais LB, Dykes J, Anderson P, Whitin JC, Keyserling HL, Carlone GM: Standardization of an opsonophagocytic assay for the measurement of functional antibody activity against
Streptococcus pneumoniae using differentiated HL-60 cells. Clin Diagn Lab Immunol 1997, 4:415-422.

23. Kim KH, Yu J, Nahm MH: Efficiency of a pneumococcal opsonophagocytic killing assay improved by multiplexing and by coloring colonies. Clin Diagn Lab Immunol 2003, 10:616-621.

24. Burton RL, Nahm MH: Development of a fourfold multiplexed opsonophagocytosis assay for pneumococcal antibodies against additional serotypes and discovery of serological subtypes in Streptococcus pneumoniae serotype 20. Clin Vaccine Immunol 2012, 19:835-841.

25. Ko KS, Baek JY, Song JH: Multidrug-Resistant Streptococcus pneumoniae Serotype 6D Clones inSouth Korea. J Clin Microbiol 2012, 50:818-822.

26. Carvalho Mda G, Pimenta FC, Gertz RE Jr, Joshi HH, Trujillo AA, Keys LE, Findley J, Moura IS, Park IH, Hollingshead SK, Pilishvili T, Whitney CG, Nahm MH, Beall BW, Active Bacterial Core Surveillance Team: PCR-based quantitation and clonal diversity of the current prevalent invasive serogroup 6 pneumococcal serotype, 6C, in the United States in 1999 and 2006 to 2007. J Clin Microbiol 2009, 47:554-559.

27. Lee $\mathrm{H}, \mathrm{Nahm} \mathrm{MH}$, Burton $\mathrm{R}$, Kim $\mathrm{KH}$ : Immune response in infants to the heptavalent pneumococcal conjugate vaccine against vaccine-related serotypes 6A and 19A. Clin Vaccine Immunol 2009, 16:376-381.

28. Lee HJ, Park SE, Kim KH: Immune response to 19A serotype after immunization of $19 \mathrm{~F}$ containing pneumococcal conjugate vaccine in Korean children aged 12-23 months. Korean J Pediatr 2011, 54:163-168.

29. Yu X, Gray B, Chang S, Ward JI, Edwards KM, Nahm MH: Immunity to crossreactive serotypes induced by pneumococcal conjugate vaccines in infants. J Infect Dis 1999, 180:1569-1576.

30. Lozano F, Rada C, Jarvis JM, Milstein C: Affinity maturation leads to differential expression of multiple copies of a kappa light-chain transgene. Nature 1993, 363:271-273.

31. Hackett J Jr, Rogerson BJ, O'Brien RL, Storb U: Analysis of somatic mutations in kappa transgenes. J Exp Med 1990, 172:131-137.

32. Henckaerts I, Goldblatt D, Ashton L, Poolman J: Critical differences between pneumococcal polysaccharide enzyme-linked immunosorbent assays with and without $22 \mathrm{~F}$ inhibition at low antibody concentrations in pediatric sera. Clin Vaccine Immunol 2006, 13:356-360.

33. Kim NH, Lee J, Lee SJ, Lee H, Kim KH, Park SE, Lee HJ: Immunogenicity and safety of pneumococcal 7-valent conjugate vaccine (diphtheria CRM (197) protein conjugate; Prevenar) in Korean infants: differences that are found in Asian children. Vaccine 2007, 25:7858-7865.

34. Yeh SH, Gurtman A, Hurley DC, Block SL, Schwartz RH, Patterson S, Jansen KU, Love J, Gruber WC, Emini EA, Scott DA, 004 Study Group: Immunogenicity and safety of 13 -valent pneumococcal conjugate vaccine in infants and toddlers. Pediatrics 2010, 126:e493-e505.

35. Silfverdal SA, Hogh B, Bergsaker MR, Skerlikova H, Lommel P, Borys D, Schuerman L: Immunogenicity of a 2-dose priming and booster vaccination with the 10-valent pneumococcal nontypeable Haemophilus influenzae protein D conjugate vaccine. Pediatr Infect Dis J 2009, 28:e276-e282

doi:10.1186/1471-2334-13-474

Cite this article as: Lee et al:: The 7-valent pneumococcal conjugate vaccine elicits cross-functional opsonophagocytic killing responses to Streptococcus pneumoniae serotype 6D in children. BMC Infectious Diseases 2013 13:474.

\section{Submit your next manuscript to BioMed Central and take full advantage of:}

- Convenient online submission

- Thorough peer review

- No space constraints or color figure charges

- Immediate publication on acceptance

- Inclusion in PubMed, CAS, Scopus and Google Scholar

- Research which is freely available for redistribution 